\title{
Urgences
}

\section{Carré noir sur fond noir}

\section{Jean-Marc Bélanger}

Numéro 27, mars 1990

Images imaginaires

URI : https://id.erudit.org/iderudit/025570ar

DOI : https://doi.org/10.7202/025570ar

Aller au sommaire du numéro

Éditeur(s)

Urgences

ISSN

0226-9554 (imprimé)

1927-3924 (numérique)

Découvrir la revue

Citer ce document

Bélanger, J.-M. (1990). Carré noir sur fond noir. Urgences, (27), 31-33.

https://doi.org/10.7202/025570ar d'utilisation que vous pouvez consulter en ligne.

https://apropos.erudit.org/fr/usagers/politique-dutilisation/ 
FRACTION DU BLANC.LA DERELICTION SE STRIE DE VAGUES ENTRELACS

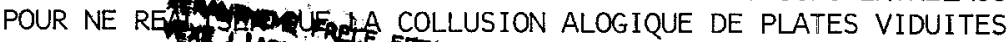

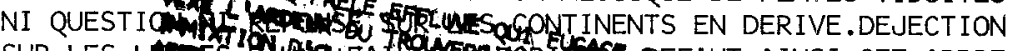

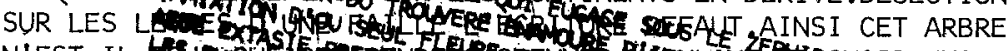

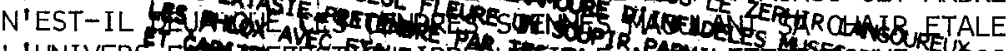

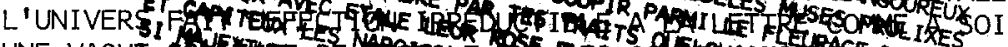

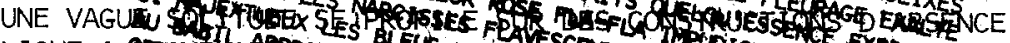

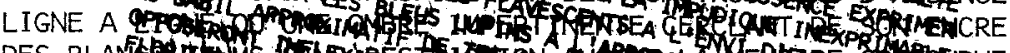
DES BLAN EN MAIN H

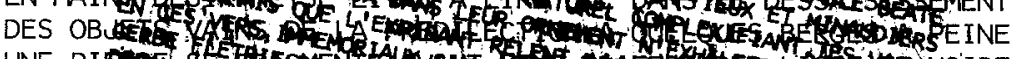

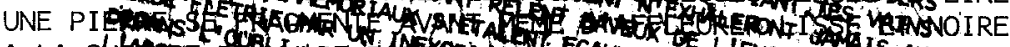

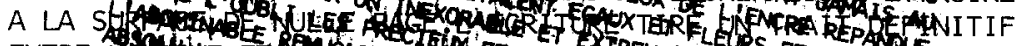

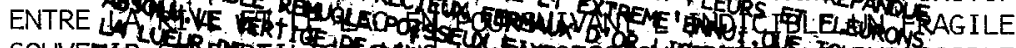

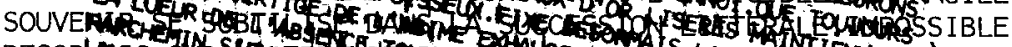

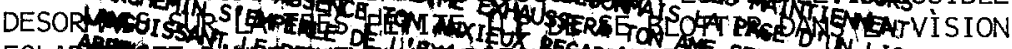

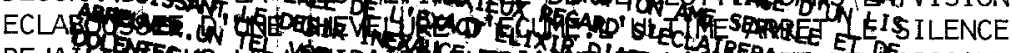

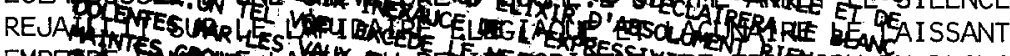
EMPERT

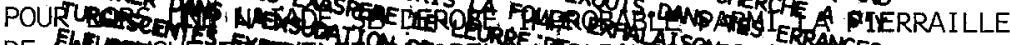

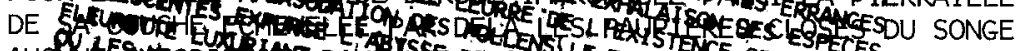

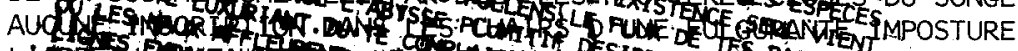

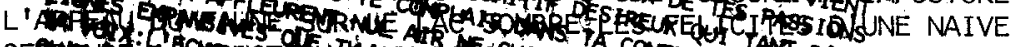

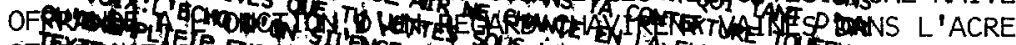

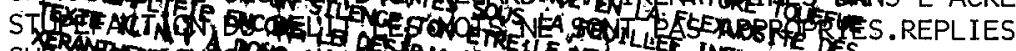

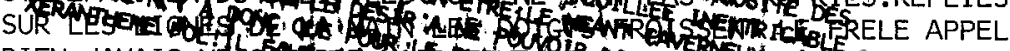

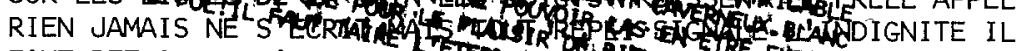
FAUT RETRAITER L'ESPACE PARQELTA DS FONDRE LA LIGNE. INFRACTION AU BLANC.JUSH UATFETWE SE RESOLVE DANS LE CALME L'OPPOSITION ENTRE LA PIERRE ET SONX INSCRIPTION JUSQU'A CE QU'ENFIN LE REGARD EMBRASSE LE LAC NOIR DE LA PAGE 
XERANTHEME FRELE. EFFLUVE QUI FUGACE SOUS LE ZEPHIR LANGOUREUX VEXE L'ARDEUR DU TROUVERE ENAMOURE D'INFIDELES MUSES PROLIXES IMMIXTION D'OU SEUL FLEURE UN SOUPIR PARMI LE FLEURAGE EXALTE AEDE EXTASIE.PRETENDRE PAR TES TRAITS QUELQU'ESSENCE EXPRIMER LES PHLOX AVEC ETALE LEUR ROSE FLA-FLA IMPUDIQUE INEXPRIMABLE ET CAPITEUX LES NARCISSES FLAVESCENTS A L'ENVI D'EXTRAVAGANCE SI MAJEXTUEUX LES BLEUS LUPINS A L'ARROGANCE INEXPIABLE BEATE AU BABIL APPROXIMATIF DE TES AGREMENTS CAPTIEUX ET MINAUDIERS OPPOSERONT INFLEXIBLES UN VIF NATUREL COMPLEXIFIANT. TES VAINS FLORILEGES SPECIEUX DANS LEUR ORNEMENT N'EXHALERONT JAMAIS AU GRAND JAMAIS QUE L'EXPIRANT RELENT BAVEUX DE L'ENCRE REPANDUE EN CES VERS IMMEMORIAUXX S'ETALENT EGAUX ET FLEURS ET FLEURONS GERBE FLETRIE PAR UN INEXORABLE ET EXTREME ENNUI.QUE TOUUOURS DEDANS L'OUBLI LES PRECIEUX FERMAUX D'OR LISERES MAINTIENNENT L'ABOMINABLE REMUGLE POISSEUX.FIXE DESORMAIS LA PAGE D'UN LIS ABSOLU.LE VERTIGE DE L'ABIME EXHAUSSERA TON AME SERVILE ET DE LA LUEUR DE L'ABSENCE TON ANXIEUX REGARD S'ECLAIRERA. LE BLANC PARCHEMIN S'EMPERLE DE L'EXACT ELIXIR D'ABSOLUMENT RIEN QUAND LANGUISSANT LE DESIR INEXAUCE DE L'EXPRESSIVITE CHERCHE A S'Y ABREUVER. UN TEL VOEU EXCEDE LE NEANT EXQUIS.DANS TES ERRANCES DOLENTES PAR LES VAUX FLEURIS LA FOLLE EXMALAISON DES ESPECES MAINTES GROUPEES EXASPERE LE LEURRE DE L'EXISTENCE.SEUL VIENT PLEURER DANS L'EXSUDATION DES POLLENS LE FLUX DE TES PASSIONS TURGESCENTES. EXPURGE L'ABYSSE PLUMITIF DESIREUX QUI TANT D'OR FLEURETTE LUXURIANT.TU TE COMPLAIS DANS TA CONTEXTURE TOUFFUE OU LES MAUX AFFLEURENT. NUL AIR NE CHANTE EN LA FLEXUOSITE DES LIGNES EXPANSIVES QUE TU JETTES SOUS LA FEUILLEE INEXTRICABLE TA VOIX:L'ECHO D'UN SILENCE.TON ETRE:LE NEANT CAVERNEUX.BLANC QU'EXEMPLIFIE ENCORE LE DESIR A NE POUVOIR LAS EN ETRE EXEMPT TEXTE IL N'Y A DONC QUE POUR LE PLAISIR DE RIEN SANS PRETEXTE XERANTHEME FOL!IL FAUT TAIRE L'ETERNITE VAINE AU POETE JALOUX 
FRACTION DU BLANC.LA DERELICTION SE STRIE DE VAGUES ENTRELACS POUR NE REALISER QUE LA COLLUSION ALOGIQUE DE PLATES VIDUITES NI QUESTION NI REPONSE SUR LES CONTINENTS EN DERIVE.DEJECTION SUR LES LEVRES D'UNE FAILLE L'ECRITURE DEFAUT.AINSI CET ARBRE N'EST-IL DEJA QUE CES CENDRES SIENNES MACULANT SA CHAIR ETALE L'UNIVERS FAIT DEFECTION IRREDUCTIBLE A LA LETTRE COMME A SOI UNE VAGUE SOLITUDE SE PROFILE SUR DES CONSTRUCTIONS D'ABSENCE LIGNE A LIGNE.QU'UNE OMBRE IMPERTINENTE CERCLANT DE SON ENCRE DES BLANCS TENUS.SANS RESTRICTION AUCUNE LA MEMOIRE CONFONDUE EN MAINT DESIR NE SE LIVRE A LIRE QUE DANS LE DESSAISISSEMENT DES OBJETS VAINS DE LA PREDILECTION.A QUELQUES BERGES A PEINE UNE PIERRE SE FRAGMENTE AVANT MEME D'AFFLEURER LISSE ET NOIRE A LA SURFACE DE NULLE PAGE. L'ECRITURE TIRE UN TRAIT DEFINITIF ENTRE LA RIVE ET LE LAC EN POURSUIVANT L'INDICIBLE. UN FRAGILE SOUVENIR SE SUBTILISE DANS LA SUCCESSION LITTERALE. IMPOSSIBLE DESORMAIS SUR LA TRES PETITE ILE DE SE BLOTTIR DANS LA VISION ECLABOUSSEE D'UNE CHEVELURE D'ECUME. L'ULTIME ARRET LE SILENCE REJAILLIT SUR LE LAPIDAIRE ELEGIAQUE ET LACUNAIRE LE LAISSANT EMPETRE DANS LE LACIS DU DELIT SCRIPTURAIRE. FRACTION DU BLANC POUR LORS UNE NAIADE SE DEROBE IMPROBABLE PARMI LA PIERRAILLE DE SA COUCHE ECHEVELEE. PAR DELA LES PAUPIERES CLOSES DU SONGE AUCUNE INSCRIPTION DANS LES CHAIRS D'UNE FULGURANTE IMPOSTURE L'APPEAU DU FAUNE SUR LE LAC SOMBRE. LES FELICITES D'UNE NAIVE OFFRANDE A L'ONCTION D'UN REGARD CHAVIRENT VAINES DANS L'ACRE STUPEFACTION DU DELIT.LES MOTS NE SONT PAS APPROPRIES.REPLIES SUR LES LIGNES DE LA MAIN LES DOIGTS FROISSENT LE FRELE APPEL RIEN JAMAIS NE S'ECRIT.MAIS TOUT REPLI SIGNALE L'INDIGNITE IL FAUT RETRAITER L'ESPACE PARCELLAIRE AGREUVER LE VIDE LITTERAL FONDRE LA LIGNE. INFRACTION AU BLANC. JUSQU'A CE QUE SE RESOLVE DANS LE CALME L'OPPOSITION ENTRE LA PIERRE ET SON INSCRIPTION JUSQU'A CE QU'ENFIN LE REGARD EMBRASSE LE LAC NOIR DE LA PAGE 